\title{
Desvios de custos e prazos em empreendimentos da construção civil: categorização e fatores de influência
}

\author{
Cost and time overrun in construction projects: \\ categorization and influencing factors
}

\author{
Elisa Atália Daniel Muianga \\ Ariovaldo Denis Granja \\ Joyce de Andrade Ruiz
}

\section{Resumo



esvios de custos e prazos são desafios recorrentes na construção civil.

Neste artigo, os autores objetivaram revisar sistematicamente a

literatura relacionada a desvios de prazos e custos para determinar uma estrutura de categorização e respectivos fatores responsáveis por esses desvios. Foi empregada a abordagem Systematic Literature Review (Revisão Sistemática de Literatura - RSL) de maneira metódica, para proporcionar acompanhamento e reprodução futura. Informações relevantes foram extraídas dos artigos selecionados e posteriormente sintetizadas para a obtenção da proposta de categorização e respectivos fatores responsáveis pelos desvios. Nove categorias foram identificadas e definidas com base em seus determinantes: relações governamentais; contratos; organização; gerenciamento; financiamento; projeto e documentação; alterações de escopo; aspectos ambientais e econômicos; e atividades e equipamentos. Além disso, 95 fatores de influência foram identificados e anexados às categorias pertinentes. Apesar de a literatura ser heterogênea em relação a contextos e regiões globais, o que dificulta a condução da RSL, houve boa sustentação para os fatores identificados. Escassez de evidências foram identificadas na América Latina, particularmente no Brasil. A literatura existente em desvios de prazos e custos até o momento se mostrou multifacetada, com 92 artigos publicados em 46 fontes diferentes no intervalo de tempo de 1985 a 2014. A maioria das pesquisas teve como foco o estudo de alguns desses fatores em particular, enquanto esta pesquisa classificou-os e sintetizou-os de forma a possibilitar uma visão nova e mais abrangente sobre o fenômeno.

Palavras-chaves: Desvios de custos. Desvios de prazos. Atrasos. Construção civil.

Elisa Atália Daniel Muianga Universidade Estadual de Campinas e Asna Construções e Engenharia Maputo - Moçambique

Ariovaldo Denis Granja Universidade Estadual de Campinas Campinas - SP - Brasil

Joyce de Andrade Ruiz Universidade Estadual de Campinas Campinas - SP - Brasil

Recebido em 08/06/14 Aceito em 18/10/14

\section{Abstract}

Cost and time overruns are recurrent challenges in construction projects. This article aims to systematically review the literature on cost and time overruns in construction projects in order to categorize and identify the influence factors that cause such overruns. A systematic literature review (SLR) was carried out in a methodical fashion to ensure it is repeatable and can be scrutinised. Relevant information was extracted from the selected articles and subsequently synthetized in order to categorise and identify the influence factors, which trigger time and cost overruns. Nine categories were identified and defined on the basis of their determinants: governmental relations; contracts; organization; management; financing; design and documentation; scope changes; environmental and economic and activities and equipment. Moreover, 95 influencing factors were identified and classified into different categories. While the literature differs with regards to different situations and global regions, complicating the RSL process, there is considerable support for these factors. Evidence scarcity was detected in Latin America, particularly in Brazil. The literature on time and cost overruns in construction is contrasting, with 92 articles from 46 different reference sources from 1985 to 2014. Most of the research focused on specific influence factors, while this study categorised and synthesised them in order to provide a novel and broader perspective of the phenomenon.

Keywords: Costoverrun. Time overrun. Delays. Construction industry. 


\section{Introdução}

Custos e prazos estão entre as principais restrições em empreendimentos de construção civil. Essas restrições se estabelecem no sentido de não se poder exceder o orçamento e o prazo propostos para dado empreendimento. Por outro lado, ainda persiste uma percepção intuitiva e fragmentada sobre os fatores que influenciam a ocorrência de desvios de custos e prazos em empreendimentos do setor da construção civil. O agrupamento desses fatores discretos em categorias pode auxiliar usuários desse tipo de informação a eliminar o que é irrelevante ou o que não interessa, possibilitando guiar a atenção para aquilo que mais importa. Além disso, categorizações e organização de informação são úteis para possibilitar uma visão mais geral sobre assuntos complexos (FELDMAN, 2004).

Um volume considerável de estudos primários sobre desvios de custos e prazos em empreendimentos de construção civil foi publicado. De acordo com a Revisão Sistemática de Literatura (RSL) realizada nesta pesquisa, a temática sobre desvios de custos e prazos em empreendimentos da construção civil continua recebendo atenção da comunidade científica global, o que remete à constatação de que esse problema ainda é recorrente e desafiador no setor. Tais estudos discutiram os fatores de influência que causam esses desvios e abrangem grande diversidade geográfica e contextual. Entretanto, poucos deles se propuseram a categorizar e a classificar os fatores que influenciam os desvios, de forma a propiciar uma compreensão mais holística sobre o assunto.

Especificamente no Brasil, foram encontrados poucos estudos primários com o devido rigor científico nessa temática. Como exemplo, Ricardino, Silva e Alencar (2013) apresentaram, em sua pesquisa, as causas mais frequentes de reivindicações em contratos administrativos para execução de empreendimentos de infraestrutura e obras e serviços de grande complexidade técnica no Brasil. Outro artigo, porém de cunho não científico, foi produzido pela mídia especializada em relação às causas de desvios em obras de infraestruturas da copa do mundo de futebol de 2014 (BOMBIG; GORCZESKI, 2013).Tendo em vista o nível atual de atividade do setor e os relatos recorrentes na imprensa sobre a ocorrência de desvios de custos e prazos em empreendimentos nacionais, urgem mais estudos científicos na temática.
O objetivo desta pesquisa foi revisar sistematicamente a literatura sobre desvios de prazos e custos em empreendimentos da construção civil, de forma a produzir uma estrutura de categorização e respetivos fatores responsáveis por esses desvios.A proposta de categorização e levantamento sistematizado de fatores oferecidos nesta pesquisa pode auxiliar a diagnosticar aqueles mais relevantes caso a caso, servindo de base para a adoção de estratégias de tomada de decisão e visando à maior conformidade de custos e prazos inicialmente planejados em empreendimentos do setor.

\section{Método de pesquisa}

Systematic Literature Review (Revisão Sistemática de Literatura - RSL) é um estudo secundário pelo qual é possível identificar, avaliar e interpretar as pesquisas disponíveis em estudos primários, relevantes para uma questão de pesquisa específica (KITCHENHAM et al., 2009). O objetivo de uma RSL é extrair detalhes específicos de artigos já publicados de pesquisas pertinentes ao tema (BRERETON et al., 2007). Destina-se à coleta de informações (evidências) que embasem as pesquisas em desenvolvimento, na qual o pesquisador procura identificar e integrar a melhor evidência de pesquisa disponível com domínio de expertise (KITCHENHAM et al., 2009). Além disso, as RSLs permitem novos desdobramentos a partir de estudos primários já publicados, uma vez que a síntese desse conjunto pode proporcionar novas inferências, as quais as pesquisas, individualmente, não são capazes de oferecer.

As RSLs sobre as evidências disponíveis na literatura têm sido pouco exploradas na construção civil; de outra forma, poderiam fornecer percepções e orientações para intervenções nas necessidades operacionais de profissionais e de agentes públicos (TRANFIELD; DENYER; SMART, 2003). Essa é uma técnica científica eficiente e menos dispendiosa quando comparada à produção de novos estudos primários, além de obter resultados mais precisos com melhor reflexo da realidade (MULROW, 1994). Estudos secundários como as RSLs podem ser considerados como estratégias direcionadas a pesquisas pragmáticas em diversas áreas do conhecimento, com o intuito de contemplar tanto as comunidades acadêmicas como as profissionais de uma área. Entretanto, para o desenvolvimento da RSL, existe um conjunto de estágios a serem seguidos (Quadro 1). 
Quadro 1 - Estágios e etapas para condução da RSL

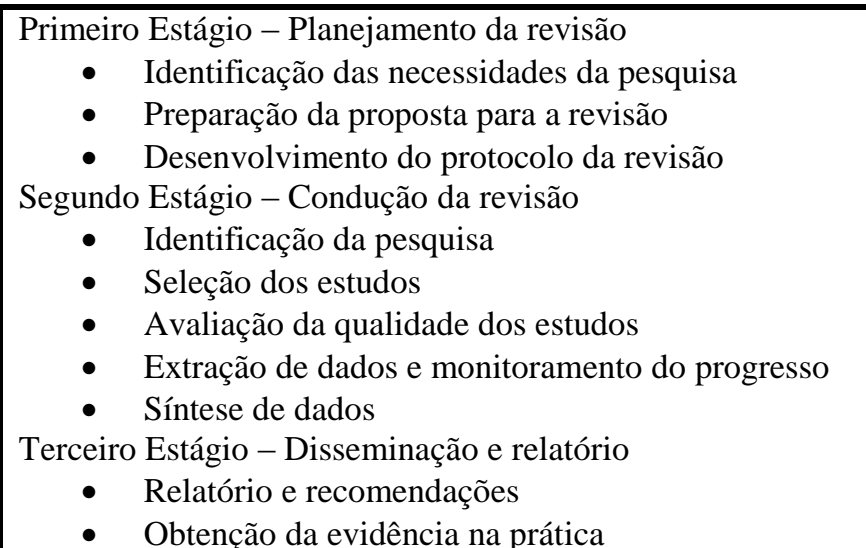

Fonte: Kitchenham et al. (2009).

A seguir, apresentam-se as descrições dos estágios e as respectivas etapas realizadas nesta pesquisa.

\section{Planejamento da revisão}

A literatura estudada apresentou grande diversidade no entendimento de conceitos e terminologias. Portanto, tornou-se necessário apresentar um alinhamento desses termos e definições para a condução desta pesquisa. Assim, oferece-se uma contribuição adicional, para que esses conceitos e terminologias possam ser tratados de maneira consistente.

As pesquisas analisadas trazem diferentes percepções sobre os desvios de custos. O conjunto das definições a seguir dá origem ao termo "desvio de custos", utilizado nesta pesquisa:

(a) "A diferença entre o custo real e o estimado, expressa como uma percentagem do custo estimado, considerando-se todos os custos a valores constantes"1 (LEE, 2008);

(b) "A diferença percentual do custo final do projeto e o custo de adjudicação do contrato" (ROWLAND, 1981);

(c) "O excesso do custo real acima do orçamento. Ele é também por vezes chamado de 'escalação de custos', 'aumento de custos' ou 'excedência do orçamento"” (ZHU; LI, 2004).

De maneira semelhante, a definição sobre atrasos na construção civil contemplou diversas visões para o que nesta pesquisa se convencionou chamar de "desvio de prazos", conforme segue:

(a) "O tempo após a data de conclusão especificada no contrato" (RAMANATHAN; NAKAYANAN; IDRUS, 2012; FUGAR;

${ }^{1}$ Ao que tudo indica, o autor se refere a valores descontados a determinada taxa de desconto (juros) a um ponto específico do tempo.
AGYAKWAH-BAAH, 2010; NAJAFABADI; PIMPLIKAR, 2013; JAHANGER, 2013);

(b) "A diferença entre a duração do prazo de contrato original do empreendimento antes do seu início e seu prazo real apurado" (VIDALIS; NAJAFI, 2002);

(c) "O atraso além da conclusão prevista da data rastreável pelo contratante" (KAMING et al., 1997a).

Fatores de influência são aqui definidos como as causas responsáveis pela ocorrência de desvios de custos e prazos em empreendimentos. Esses fatores podem ser agrupados de acordo com as similaridades das variáveis que eles apresentam. Cada agrupamento compõe uma categoria, que é considerada como um grupo de fatores que exprimem diversas relações entre ideias ou fatos relacionados que caracterizam e identificam os problemas mais predominantes. Essas categorias são definidas, com base em seus determinantes (descritores), mais à frente, no Quadro 4.

\section{Categorias e determinantes identificados na RSL}

A partir das 92 pesquisas da RSL, 6 delas apresentaram propostas de categorizações com os principais fatores causadores de desvios de prazos e custos (LONG et al., 2004; ASSAF; ALKHALIL; AL-HAZMI, 1995; LE-HOAI; LEE; LEE, 2008; NUTAKOR, 2007; GONZÁLEZ et al., 2014; SUGIHARTO; HAMPSON, 2003), que são descritas na forma de seus determinantes.

Long et al. (2004):

(a) financiador (fundos do empreendimento);

(b) proprietário (liderança, responsabilidade, processo de licitação); 
(c) construtor (experiência, gerenciamento, produtividade);

(d) consultor (experiência, assistência no projeto e no empreendimento);

(e) atributos do projeto (escopo do projeto ambíguo, omissões e erros);

(f) coordenação (comunicação e compreensão); e

(g) ambiental (efeitos climáticos e condições do solo).

Assaf, Al-Khalil e Al-Hazmi (1995):

(a) financiamento (fundos para o empreendimento);

(b) alterações (estudo de viabilidade e análise do empreendimento);

(c) planejamento e cronograma (cronograma e aprovação de recursos e demais requisitos);

(d) mão de obra (nacionalidade dos trabalhadores, demanda e qualificação);

(e) material (especificações, aquisição e armazenamento);

(f) ambiental (efeitos climáticos, sociais e culturais); e

(g) relações governamentais (permissões, burocracia e regulamentos).

Le-Hoai, Lee e Lee (2008):

(a) proprietário (pagamentos e dificuldades financeiras);

(b) construtor (fundos, gerenciamento e experiência);

(c) consultor (experiência, assistência no projeto e no empreendimento);

(d) projeto (alterações);

(e) material/trabalho (aquisição e habilidade dos trabalhadores); e

(f) externo (condições climáticas e regulamentações ambientais).

Nutakor (2007):

(a) definição do escopo (diferença de opiniões, definição de responsabilidades, riscos), reivindicações e pleitos contratuais (magnitude e gestão das alterações);

(b) riscos de contrato (diferenças na interpretação);

(c) questões de gestão de riscos (experiência, avaliação das dificuldades do trabalho);

(d) condições do mercado (baixos níveis salariais e avaliação da mão de obra); e (e) processos de licitação (requisições para a licitação e omissões nesse processo).

González et al. (2014):

(a) projeto (concepção e documentação);

(b) planejamento (programação e planejamento);

(c) subempreiteiros (produtividade $\mathrm{e}$ coordenação);

(d) execução do trabalho (produtividade e métodos implementados);

(e) material e equipamento (provisionamento); e

(f) clima (condições climáticas).

Sugiharto e Hampson (2003):

(a) projeto e documentação (concepção, qualidade na documentação apresentada, especificações e revisão do projeto);

(b) profissional de gerenciamento (planejamento, cronograma e coordenação);

(c) pessoas (habilidades e inspeção);

(d) execução (métodos implementados, equipamento e horas extras);

(e) material (qualidade, manuseio, provisionamento e armazenamento); e

(f) externo (condições climáticas e do solo).

Pode-se perceber que as terminologias e conceitos utilizados nessas seis pesquisas foram diversificados e heterogêneos. Esse fato exigiu uma análise detalhada sobre os entendimentos de cada autor e uma decorrente proposta de homogeneização para fins de consolidação da terminologia nesta pesquisa. A contribuição de categorizações e respetivos fatores é apresentada mais à frente neste artigo.

\section{Condução da revisão}

Para a realização da RSL, foram identificadas as palavras-chave com vistas à seguinte questão da pesquisa: "quais são os fatores responsáveis por desvios de custos e prazos em empreendimentos da construção civil?". As palavras-chave que caracterizam o tema investigado são "atraso" ou "desvios de prazo", como time overrun, e "desvios de custos", como cost overrun. Assim sendo, essas palavras foram adotadas e utilizadas nas bases de dados para a elaboração do referencial teórico.

Para restringir a busca e dar ênfase apenas para o setor da construção civil, foi adicionada a palavra "construção", construction. É importante ressaltar que a pesquisa não considerou eventuais casos de desvios de prazos e custos abaixo dos inicialmente planejados, ou seja, ocorrências de time 
underrune/ou cost underrun, pois fugiriam ao escopo proposto.

As bases de dados utilizadas para a busca da literatura foram a Web Of Knowledge, Science Direct e Scopus. A base de dados Scielo também foi utilizada para a busca, bem como termos em português e suas variações, para possível inclusão de estudos científicos nacionais ou publicados nessa língua. Mesmo aplicados esses critérios, não foram encontradas referências nacionais. Ainda assim, os autores optaram por incluir duas fontes nacionais, consideradas relevantes para responder à questão de pesquisa formulada:

(a) Ricardino, Silva e Alencar (2013), pesquisa de cunho científico; e

(b) Bombig e Gorczeski (2013), pesquisa proveniente da mídia especializada.

A Tabela 1 ilustra as palavras-chave utilizadas, a soma dos trabalhos obtidos nas três bases de dados durante a busca e o processo de filtragem utilizado.

Com a aplicação das palavras-chave, foram obtidos 7.218 trabalhos, distribuídos em suas bases de dados. Para a aplicação do primeiro filtro, foram analisados os temas dos trabalhos. Os temas que não se relacionavam com construção civil, prazos, custos e gerenciamento foram excluídos, o que resultou em 6.015 trabalhos não relevantes para o tema.

O segundo filtro consistiu em analisar as duplicidades. Os 1.203 trabalhos aparentemente relevantes foram analisados em conjunto. Observou-se que determinadas pesquisas foram indexadas em mais de uma base de dados; com isso, foram excluídos outros 524 trabalhos.

Para as 679 pesquisas restantes, a análise foi mais aprofundada. $\mathrm{O}$ terceiro filtro foi aplicado na forma de leitura dos resumos. Por existirem resumos curtos, que dificultavam a compreensão da essência do trabalho, também foram analisados os objetivos, resultados e as principais conclusões. Com este filtro, apenas 76 trabalhos apresentaram relevância para a resposta à questão de pesquisa formulada.

Com a leitura das 76 pesquisas aderentes, aplicouse o quarto filtro. A obtenção de trabalhos a partir do referencial de pesquisas aderentes é descrita como "Amostragem bola de neve" (ABN)
(Snowball Sampling) (BIERNACKI; WALDORF, 1981) e caracterizou o quarto filtro aplicado. A RSL e a ABN são métodos característicos do paradigma baseado em evidências (Evidence-based Paradigm). A ABN consiste em buscar outros estudos citados dentro das referências da própria RSL, considerando adequação temática e frequência de citação. É particularmente aplicada para garantir que referências de pesquisas seminais estejam dentro da amostra (BIERNACKI; WALDORF, 1981). Com a ABN, totalizaram-se 92 artigos aderentes, que compuseram a RSL em conjunto com a ABN.

Entretanto, as pesquisas não tiveram o mesmo tipo de abordagem. Em sua maioria, foi utilizado o método Survey com questionários abertos e estruturados para a coleta de dados. Um pequeno número de trabalhos apresentou debates abertos como outra opção de coleta de dados. Contudo, o maior objetivo das pesquisas era apurar os fatores que mais contribuíam para a ocorrência de desvios de custos e prazos e para o gerenciamento inadequado nos empreendimentos. Esse aspecto orientou as decisões sobre inclusão e exclusão dos artigos aderentes e a apuração dos fatores mais relevantes apresentados em cada pesquisa. A Figura 1 mostra as pesquisas aderentes de acordo com as bases de dados onde elas estão indexadas.

Com a leitura das pesquisas aderentes, foi possível analisar em que região elas tinham sido realizadas. A Figura 2 mostra que a maior parte dos estudos foi realizada na Ásia e, em seguida, na África. A Europa segue como terceira região com um número significativo de pesquisas publicadas, enquanto a região da Oceania e América apresentam menos trabalhos publicados relacionados com o tema.

A Tabela 2 demonstra as fontes acompanhadas das respetivas quantidades de pesquisas publicadas em relação aos desvios de custos e prazos em empreendimentos de construção civil.

Então, elaborou-se uma síntese narrativa das 92 pesquisas, que buscou reunir os principais resultados dos estudos incluídos na RSL (PETTICREW, 2009). A partir de uma visão de síntese dessas pesquisas, analisaram-se as causas de desvios de custos e prazos, e foi proposta uma estrutura de categorização e os respectivos fatores de influência. 
Tabela 1 - Palavras-chave utilizadas nas bases, número resultante de trabalhos e processo de filtragem

\begin{tabular}{|c|c|c|}
\hline \multicolumn{2}{|r|}{ Base de dados (Web Of Knowledge, Science Direct e Scopus) } & $\begin{array}{c}\text { Trabalhos } \\
\text { encontrados }\end{array}$ \\
\hline \multirow{4}{*}{  } & cost overrun and time overrun and construction & 3.718 \\
\hline & cost overrun and time overrun and civil construction & 1.164 \\
\hline & $\begin{array}{l}\text { cost overrun and time overrun and construction and } \\
\text { (enablers or variables or influencing factors or framework) }\end{array}$ & 1.794 \\
\hline & $\begin{array}{l}\text { cost overrun and time overrun and construction and } \\
\text { developing countries }\end{array}$ & 542 \\
\hline \multicolumn{2}{|c|}{ Total das pesquisas ainda sem filtros } & 7.218 \\
\hline \multirow{4}{*}{$\underset{\text { 莺 }}{\stackrel{n}{1}}$} & $1^{\circ}$ (Leitura dos temas - menos 6.015 pesquisas) & 1.203 \\
\hline & $2^{\circ}$ (Sem duplicidades - menos 524 pesquisas) & 679 \\
\hline & $3^{\circ}$ (Leitura do resumo - menos 603 pesquisas) & 76 \\
\hline & $4^{\circ}$ (Amostragem bola de neve(ABN) - mais 16 pesquisas) & 16 \\
\hline \multicolumn{2}{|c|}{ Total das pesquisas aderentes } & 92 \\
\hline
\end{tabular}

Figura 1- Análise das pesquisas aderentes de acordo com a respectiva base de dados



Figura 2- Análise da distribuição global do percentual de quantidade de pesquisas publicadas sobre desvios de custos e prazos

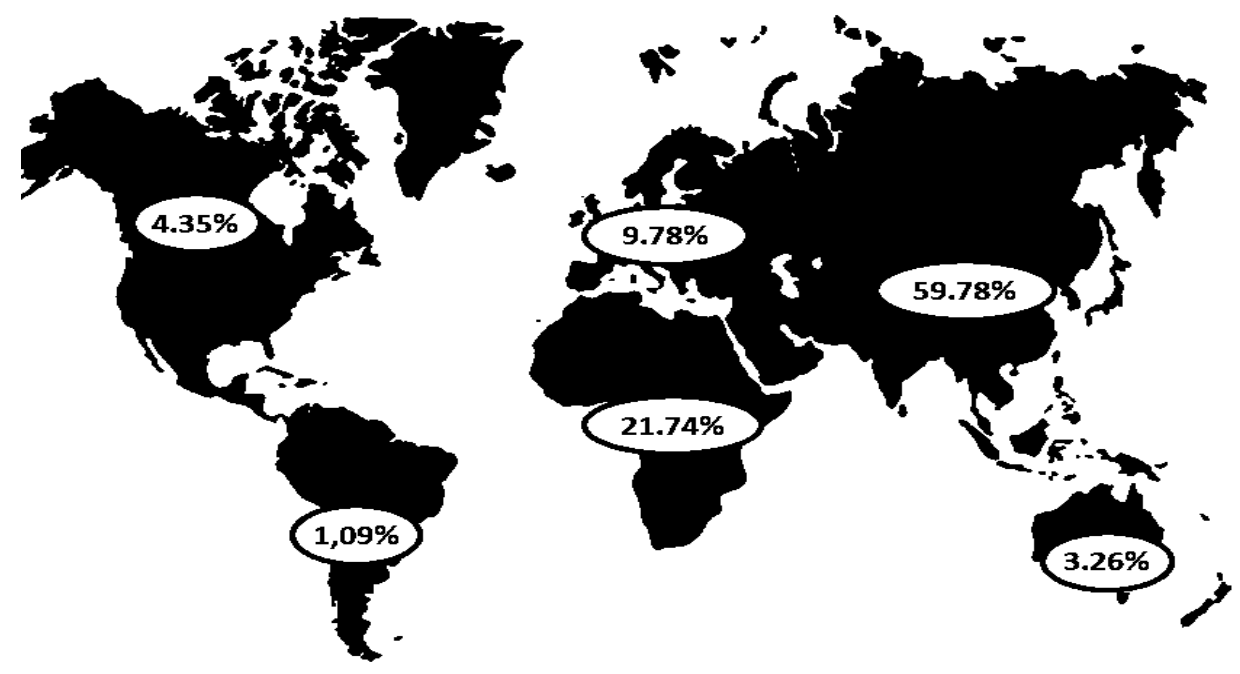

84 Muianga, E. A. D.; Granja, A. D.; Ruiz, J. de A. 
Tabela 2 - Análise das pesquisas de acordo com a fonte, autores e ano de publicação (Continua)

\begin{tabular}{|c|c|c|}
\hline $\begin{array}{c}\mathrm{N}^{0} \text { de } \\
\text { trabalhos }\end{array}$ & Título de jornal, conferência & Autores e ano de publicação \\
\hline 20 & International Journal of Project Management & $\begin{array}{l}\text { Akintoye e MacLeod (1997); Al-Momani } \\
\text { (2000); Arditi, Akan e Gurdamar (1985); } \\
\text { Assaf e Al-Hejji (2006); Chan e } \\
\text { Kumaraswamy (1997); Doloi et al. (2012); } \\
\text { Fallahnejad (2013); Frimpong, Oluwoye e } \\
\text { Crawford (2003); Iyer e Jha (2005); Kaliba, } \\
\text { Muya e Mumba (2009); Kaming et al. } \\
\text { (1997b); Dlakwa e Culpin (1990); } \\
\text { Manavazhi e Adhikari (2002); Mansfield, } \\
\text { Ugwu e Doran (1994); Odeh e Battaineh } \\
\text { (2002); Ogunlana, Promkuntong e } \\
\text { Jearkjirm (1996); Sambasivan e Soon } \\
\text { (2007); Sweis et al. (2008); Wang e Yuan } \\
\text { (2011); Zou, Zhang e Wang (2007). }\end{array}$ \\
\hline 9 & Journal of Construction Engineering and Management & $\begin{array}{l}\text { Abd El-Razek, Bassioni e Mobarak (2008); } \\
\text { Elinwa e Buba (1993); González et al. } \\
\text { (2014); Iyer e Jha (2006); Lo, Fung e Tung } \\
\text { (2006); Love } \text { et al. (2010); Okpala e } \\
\text { Aniekwu (1988); Rosenfeld (2014); } \\
\text { Semple, Hartman e Jergeas (1994). }\end{array}$ \\
\hline 3 & Journal of Management in Engineering & $\begin{array}{l}\text { Assaf, Al-Khalil e Al-Hazmi (1995); } \\
\text { Mahamid, Bruland e Dmaidi(2012); Shane } \\
\text { et al. (2009). }\end{array}$ \\
\hline 3 & Construction Management and Economics & $\begin{array}{l}\text { Kaming (1997a); Koushki, Al-Rashid e } \\
\text { Kartam(2005); Olawale e Sun (2010) }\end{array}$ \\
\hline 3 & Procedia Engineering & $\begin{array}{l}\text { Hamzahet al. (2011); Orangi, } \\
\text { Palaneeswaran e Wilson(2011); Soekimanet } \\
\text { al. (2011). }\end{array}$ \\
\hline 3 & $\begin{array}{l}\text { Journal of Financial Management of Property and } \\
\text { Construction }\end{array}$ & $\begin{array}{l}\text { Dominic e Smith (2014); Shehu, Endut e } \\
\text { Akintoye(2014); Adnan, Jomah e Mohan } \\
(2009) .\end{array}$ \\
\hline 2 & $\begin{array}{l}\text { Australasian Journal of Construction Economics and } \\
\text { Building }\end{array}$ & $\begin{array}{l}\text { Fugar e Agyakwah-Baah, (2010); Mahamid } \\
(2013) \text {. }\end{array}$ \\
\hline 2 & $\begin{array}{l}\text { Engineering, Construction and Architectural } \\
\text { Management }\end{array}$ & $\begin{array}{l}\text { Alaghbariet al. (2007); Koushki e Kartam } \\
\text { (2004). }\end{array}$ \\
\hline 2 & Journal of Engineering, Design and Technology & Mañelele e Muya (2008); Oladapo (2007). \\
\hline 2 & Building and Environment & $\begin{array}{l}\text { Chan e Kumaraswamy (1996); Ogunlana e } \\
\text { Olomolaiye (1989). }\end{array}$ \\
\hline 2 & Alexandria Engineering Journal & Aziz (2013a, 2013b). \\
\hline 2 & KSCE Journal of Civil Engineering & $\begin{array}{l}\text { Le-Hoai, Lee e Lee (2008); Ribeiro et al. } \\
\text { (2013). }\end{array}$ \\
\hline 1 & Automation in Construction & Josephson e Hammarlund (1999). \\
\hline 1 & Australian Journal of Basic and Applied Sciences & Jahanger (2013). \\
\hline 1 & Built Environment Project and Asset Management & Park e Papadopoulou (2012). \\
\hline 1 & Cost Engineering (Morgantown, West Virginia) & Nutakor (2007). \\
\hline 1 & $\begin{array}{l}\text { European Journal of Civil Engineering and } \\
\text { Architecture }\end{array}$ & Murray e Seif (2013). \\
\hline 1 & Habitat International & Hwang, Zhao e NG (2013). \\
\hline 1 & Humanity \& Social Sciences Journal & Haq e Aslam (2013). \\
\hline
\end{tabular}


Tabela 2 - Análise das pesquisas de acordo com a fonte, autores e ano de publicação (Continuação)

\begin{tabular}{|c|c|c|}
\hline 2 & International Journal of Construction Management & $\begin{array}{l}\text { Enshassi, Kumaraswamy e Jomah (2010); } \\
\text { Muya et al. (2013). }\end{array}$ \\
\hline 1 & International Journal of Engineering and Technology & Pourrostam e Ismail (2012). \\
\hline 1 & International Journal of Project Management & Long et al. (2004). \\
\hline 1 & $\begin{array}{l}\text { International Journal of Sustainable Construction } \\
\text { Engineering and Technology }\end{array}$ & Memon, Rahman e Azis (2011). \\
\hline 1 & Journal of Civil Engineering and Management & Enshassi, Arain e Al-Raee (2010). \\
\hline 1 & Journal of Civil \& Environmental Engineering & Ahomidan (2013). \\
\hline 1 & Journal of Applied Sciences & Rahman, Memon e Karim (2013a). \\
\hline 1 & Journal of Advanced Research & Marzouk e El-Rasas (2014). \\
\hline 1 & Journal of Mechanical and Civil Engineering & Najafabadi e Pimplikar (2013). \\
\hline 1 & Journal of Urban Planning and Development & Lee (2008). \\
\hline 1 & $\begin{array}{l}\text { Journal of the South African Institution of Civil } \\
\text { Engineering }\end{array}$ & Kamanga e Steyn (2013). \\
\hline 1 & Modern Applied Science & Rahman, Menon e Karim (2013b). \\
\hline 1 & Science and Technology Foundation, in Portuguese & Couto (2006). \\
\hline 1 & Structural Survey & Kadir et al. (2005). \\
\hline 1 & Transportation & Makovšek, Tominc e Logožar (2012). \\
\hline 4 & Thesis & $\begin{array}{l}\text { Al-Najjar (2008); Creedy (2006); Nega } \\
\text { (2008); Roachanakanan (2005). }\end{array}$ \\
\hline 2 & $\begin{array}{l}\text { ARCOM } 2010 \text { Proceedings of the 26th Annual } \\
\text { Conference }\end{array}$ & Motaleb e Kishk (2010); Sunday (2010). \\
\hline 1 & $\begin{array}{l}\text { An Empirical Study. ASC Proceedings of the 39th } \\
\text { Annual Conference }\end{array}$ & Ahmedet al.(2003). \\
\hline 1 & $\begin{array}{l}\text { Proceedings from the International Conference on } \\
\text { Advances in Engineering and Technology }\end{array}$ & Alinaitwe,Mwakali e Hansson (2006). \\
\hline 1 & $\begin{array}{l}\text { CME } 25 \text { Conference construction management and } \\
\text { economics "past, present and future" }\end{array}$ & Pires, Teixeira e Moura (2007). \\
\hline 1 & National Postgraduate Conference (NPC), 2011 & Potty, Irdus e Ramanathan (2011). \\
\hline 1 & $\begin{array}{l}2012 \text { IEEE Colloquium on Humanities, Science and } \\
\text { Engineering (CHUSER) }\end{array}$ & Rahman et al. (2012). \\
\hline 1 & $\begin{array}{l}\text { The IEEE International Conference On Industrial } \\
\text { Engineering And Engineering Management }\end{array}$ & Ramanathan, Potty e Idrus (2011). \\
\hline 1 & $\begin{array}{l}\text { ARCOM } 2008 \text { Proceedings of the } 24 \text { th Annual Arcom } \\
\text { Conference }\end{array}$ & Ren, Atout e Jones (2008). \\
\hline 1 & $\begin{array}{l}\text { Proceedings The 9th East Asia-Pacific Conference on } \\
\text { Structural Engineering and Construction. }\end{array}$ & Sugiharto e Hampson (2003). \\
\hline 1 & $\begin{array}{l}\text { 4th transportation specially conference of the } \\
\text { Canadian Society for civil Engineering, Montreal, } \\
\text { Quebec }\end{array}$ & Vidalis e Najafi (2002). \\
\hline 1 & $\begin{array}{l}\text { ARCOM } 2005 \text { - Proceedings of the } 21 \text { st Annual } \\
\text { Conference. }\end{array}$ & Wiguna e Scott (2005). \\
\hline
\end{tabular}

\section{Disseminação e relatório}

Neste estágio, extraíram-se os principais resultados primários dos estudos identificados na RSL. Primeiro, foi necessário definir os termos utilizados para o agrupamento dos resultados. Posteriormente, cada agrupamento de fatores compôs uma categoria, a qual caracteriza e identifica os problemas mais predominantes nela.

Posteriormente, efetuou-se uma análise das terminologias dos fatores utilizados nas diversas pesquisas, pois vários autores empregaram diferentes formas para descrever o mesmo fator. Para isso, foi realizada uma estratificação e homogeneização dos fatores de mesmo significado, agrupados dentro das categorias.

\section{Dados bibliométricos}

Os 92 trabalhos aderentes foram submetidos a análises bibliométricas. A Figura 3 apresenta a distribuição das pesquisas e os respectivos anos de publicação.

A partir da Figura 3, é possível inferir que, apesar de já existirem várias pesquisas relacionadas a desvios de custos e prazos, esse é um tema que continua a receber destaque na literatura. Observase que, a partir de 2005, há um aumento no número de pesquisas. Nos anos seguintes, esse número 
manteve a tendência de aumento;em 2013, o valor foi três vezes maior em relação a 2005. Possivelmente, até o início de 2015 poderão surgir mais pesquisas relacionadas ao tema, uma vez que este estudo foi realizado em meados de 2014. Portanto, as pesquisas sobre desvios de custos e prazos em empreendimentos do setor ainda recebem atenção, ao que tudo indica devido à complexidade e às particularidades regionais $\mathrm{e}$ contextuais do fenômeno. As medidas mitigadoras para esses desvios nem sempre são efetivas, mesmo quando há consciência sobre o problema. A busca das causas para a ocorrência desses desvios é fundamental para sua solução.

\section{Resultados temáticos: categorização e fatores de influência}

A RSL gerou uma matriz que contém grande quantidade de informações sobre as pesquisas selecionadas. A seguir, são apresentados três resultados provenientes da organização e análise dessas informações. O primeiro é a proposta da classificação das categorias e determinantes para posterior agrupamento dos fatores. O segundo é o próprio agrupamento dos fatores nas categorias propostas. O terceiro é a análise da distribuição dessas categorias, de acordo com a quantidade de fatores apresentados.

A proposta da classificação das categorias partiu da identificação das categorias propostas por outros autores (Tabela 1). Assim, foi elaborado o Quadro 4, que apresenta nove categorias propostas e os respectivos determinantes, os quais proporcionaram o agrupamento dos fatores a partir da síntese da RSL. Os determinantes representam um grupo de causas dentro de um mesmo contexto. Por exemplo, o contexto que une a categoria "gerenciamento" pode estar representado por atrasos na entrega do material, planejamento inadequado da obra, cronograma inadequado, entre outros. Dada a complexidade de análise, as categorias não levaram em consideração as responsabilidades dos diferentes agentes nas causas determinantes.

A categorização proposta nesta pesquisa agrupou as causas apresentadas a partir de estudos individuais e proporcionou uma nova classificação a partir da síntese destes, que poderá facilitar a análise dos fatores que influenciam os desvios de custos e prazos em empreendimentos do setor.

Para as nove categorias propostas, foram distribuídos 697 fatores, de acordo com os respectivos determinantes (Quadro 3). Por existirem fatores que podem receber diferentes terminologias nas diferentes pesquisas identificadas na RSL, eles foram estratificados como forma de homogeneizar e melhor agrupar os fatores dentro das categorias. Como exemplo, na pesquisa de Murray e Seif (2013), um dos fatores críticos é a "pouca experiência do contratante". Por outro lado, Odeh e Battaineh (2002) referem-se à "inadequada experiência do contratante", o que sugere o mesmo significado de Murray e Seif (2013). Portanto, levando-se em consideração esses autores e outros que se referem a esse mesmo fator, ele está apresentado no Quadro 3 na categoria "Atividades e Equipamentos" como "Falta de habilidade e experiência da construtora e da mão de obra, falta de trabalhadores qualificados no mercado local". Sendo assim, as nomenclaturas adotadas após a estratificação e homogeneização dão ênfase ao conjunto de fatores agrupados, reduzindo-se o número de fatores de 697 para 95. É importante ressaltar que algumas categorias poderiam ter sido aglutinadas dentro de outras. No entanto, os autores optaram por manter a proposta de categorização o mais próximo possível dos padrões identificados no corpo de literatura estudado.

\section{Figura 3 - Artigos por ano de publicação}

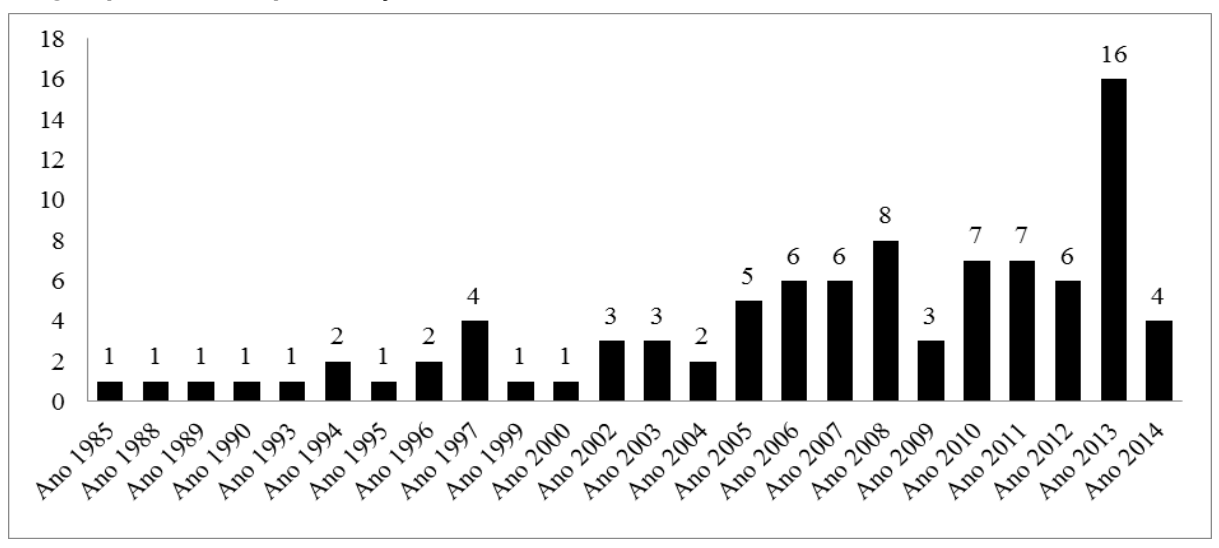


Quadro 2- Categorias de agrupamento dos fatores de desvios de custos e prazos na construção civil

\begin{tabular}{|c|c|}
\hline Categorias & Determinantes \\
\hline $\begin{array}{ll}\text { 1. } & \text { Relações } \\
\text { governamentais }\end{array}$ & $\begin{array}{l}\text { Fatores relacionados com licenças, leis, procedimentos burocráticos } \\
\text { governamentais. }\end{array}$ \\
\hline 2. Contratos & $\begin{array}{l}\text { Fatores relacionados com obrigações dos contratos, imposições } \\
\text { contratuais, contratos inadequados. }\end{array}$ \\
\hline 3. Organização & $\begin{array}{l}\text { Fatores relacionados com a supervisão, comunicação, coordenação } \\
\text { do trabalho. }\end{array}$ \\
\hline 4. Gerenciamento & $\begin{array}{l}\text { Fatores relacionados com o gerenciamento, planejamento, } \\
\text { cronograma e recursos de materiais no empreendimento. }\end{array}$ \\
\hline 5. Financiamento & Fatores relacionados ao financiamento do empreendimento. \\
\hline 6. Projeto e documentação & Fatores relacionados à qualidade do projeto. \\
\hline 7. Alterações de escopo & $\begin{array}{l}\text { Fatores relacionados ao retrabalho, ordens de variação e alteração } \\
\text { do escopo inicial. }\end{array}$ \\
\hline $\begin{array}{l}\text { 8. Aspectos ambientais e } \\
\text { econômicos }\end{array}$ & Fatores relacionados aos efeitos sociais, ambientais e econômicos. \\
\hline $\begin{array}{l}\text { 9. Atividades e } \\
\text { equipamentos }\end{array}$ & $\begin{array}{l}\text { Fatores relacionados ao desempenho, habilidades da mão de obra, } \\
\text { instruções do trabalho, métodos de construção, ferramentas e } \\
\text { equipamentos. }\end{array}$ \\
\hline
\end{tabular}

Quadro 3 - Nove categorias com os respectivos 95 fatores responsáveis por desvios de prazos e custos em empreendimentos da construção civil e quantidade de citações de cada fator (Continua)

1. RELAÇÕES GOVERNAMENTAIS - 7 agrupamentos de 14 fatores citados

Alteração de regras governamentais. (P) - 4

Aprovação dos requisitos impostos pelo governo para o empreendimento. (P/C) - 1

Burocracia no processo de licitação. (C) - 1

Insegurança política. (P/C) - 2

Pressão do governo local, situação política, obtenção de permissões governamentais legais para a construção. (P/C) - 4

Alterações de câmbio da moeda. (C) - 1

Força maior. (C) - 1

2. CONTRATOS - 13 agrupamentos de 28 fatores citados

Burocracia excessiva na organização do empreendimento causadas pelo proprietário. (P) - 1

Competição muito acirrada na fase de licitação. (P/C) - 3

Conflitos entre os participantes pelos documentos do contrato. (P/C) - 2

Duração irreal do contrato imposto pelo proprietário/cliente. (P/C) - 4

Falta de envolvimento do usuário final. (C) - 1

Falta de obtenção de seguro da obra. (NC) - 1

Imposição pela construtora de técnicas construtivas a serem utilizados no próprio contrato. (P) - 1

Modificações e alterações do contrato, questões de permissões legais e aquisições, aumento do

valor do contrato em relação ao inicialmente previsto. (P/C) - 6

Políticas internas (no âmbito do proprietário/cliente). (NC) - 1

Preço baixo de contratação. (P/C) - 3

Riscos contratuais. (P/C) - 2

Subestimação do prazo para execução do empreendimento pela construtora. $(\mathrm{P})$

Tempo insuficiente para a preparação dos documentos do empreendimento. (P/C) - 3 
Quadro 3 - Nove categorias com os respectivos 95 fatores responsáveis por desvios de prazos e custos em empreendimentos da construção civil e quantidade de citações de cada fator (Continua)

\section{ORGANIZAÇÃO - 5 agrupamentos de 35 fatores citados}

Falta de um plano para coordenação de diferentes tarefas entre diferentes subempreiteiros na execução do empreendimento. $(\mathrm{P})$ - 10

Conflito entre os participantes do empreendimento, disputas e negociações de adversidade. (P/C) - 5

Consequências negativas de decisões tomadas. (NC) - 1

Falta de conhecimento e experiência, liderança inadequada. (P/C) - 17

Relacionamento entre os trabalhadores e o gerente do empreendimento. (C) - 2

4. GERENCIAMENTO - 26 agrupamentos de 257 fatores citados

Falta de controle de tempo e dos custos de insumos. (C) - 1

Falta de planejamento e controle de custos no pré e no pós-contrato. (C) - 2

Falta de subempreiteiros e firmas especializadas para a realização de trabalhos específicos. (P/C) - 2

Falta de uso de gerenciamento da técnica de análise do valor agregado. (C) - 1

Gerenciamento insuficiente do contrato. (P/C) - 3

Gerenciamento precário do empreendimento. (P/C) - 40

Número insuficiente de encarregados para supervisão e lentidão dos serviços. $(\mathrm{P})$ - 1

Pouca comunicação e coordenação entre as equipes envolvidas no empreendimento. (P/C) - 24

Prazo insuficiente para análise correta de recursos e custos necessários para o empreendimento. (P/C) 9

Processo lento das equipes envolvidas na tomada de decisão. (P/C) - 14

Atraso na entrega do material na obra, problemas com o planejamento da entrega do material na obra.

$(\mathrm{P} / \mathrm{C})-16$

Cartelização de materiais atribuída a determinados fornecedores. (C) - 3

Escassez de material com qualidade aceitável, material inadequado, pouca oferta do material, atraso na aquisição do material importado. (P/C) - 46

Estimativa incorreta do material. (C) - 4

Falta de conhecimento em relação às disponibilidades de materiais e equipamentos pelos consultores.

(P) -2

Incremento de preço do material. (P/C) - 25

Uso de especificações inadequadas por consultores internacionais. (NC) - 1

Alteração do cronograma pelo proprietário/cliente. (P/C) - 9

Atraso na aquisição de terrenos e propriedades. (P/C) - 2

Atrasos no procedimento da execução do empreendimento. (P) - 9

Falta de comprometimento com o escopo geral do empreendimento. (P) - 1

Falta de métodos de supervisão, falta e/ou incapacidade de encarregados de supervisão. (P/C) - 10

Planejamento e cronograma inadequados. (P/C) - 20

Interrupção no fluxo de construção do empreendimento por problemas de qualidade. (P/C) - 2

Planejamento precário da logística para alocação de recursos. (P/C) - 6

Poucas frentes de serviços e preparação insuficiente de tarefas. (P/C) - 4

\section{FINANCIAMENTO - 6 agrupamentos de 78 fatores citados}

Atrasos no pagamento à construtora. $(\mathrm{P} / \mathrm{C})-11$

Custos diretos adicionais para aceleração dos trabalhos. (C) - 1

Dificuldades em relação à modalidade de pagamentos pelas agências financiadoras. $(\mathrm{P} / \mathrm{C})$ - 11

Dificuldades na quitação de financiamentos pelas construtoras. (P/C) - 1

Forma de pagamento apenas após o escopo completo do trabalho. (P/C) - 12

Problemas com o fluxo de caixa. (P/C) - 7

Problemas financeiros. (P/C) - 35

\section{PROJETO E DOCUMENTAÇÃO - 7 agrupamentos de 71 fatores citados}

Alteração do projeto. (P/C) - 21

Atraso na aprovação do projeto. (P/C) - 8

Atraso na elaboração do projeto. (C) - 1

Conflitos entre o arquiteto e os demais representantes das diferentes disciplinas do projeto. (P) - 3

Defeitos, erros e omissões no projeto. (P/C) - 34

Soluções inadequadas do projeto. (P/C) - 1

Tentativas de modificações do projeto para o alcance do padrão desejado durante a fase de construção. $(\mathrm{P} / \mathrm{C})-3$ 
Quadro 3 - Nove categorias com os respectivos 95 fatores responsáveis por desvios de prazos e custos em empreendimentos da construção civil e quantidade de citações de cada fator (Continuação)



De acordo com o Quadro 3, observa-se que 60\% dos fatores influenciam desvios tanto de prazo como de custo $(\mathrm{P} / \mathrm{C}) ; 18 \%$ dos fatores influenciaram desvios no prazo $(\mathrm{P})$; e $16 \%$, no custo (C). Apenas $6 \%$ correspondem aos fatores que não foram classificados em relação aos desvios, considerados na pesquisa como (NC). A Figura 4 apresenta uma síntese da quantidade dos fatores responsáveis pelos desvios em cada uma das nove categorias.
Com base na Figura 4,observa-se que alguns aspectos merecem reflexão. A categoria de "Gerenciamento" foi a mais relevante. Esta é uma questão de grande complexidade nos empreendimentos, uma vez que abarca a interação de todas as partes interessadas do empreendimento. Além disso, a categoria "Gerenciamento" influencia todas as demais categorizações em termos de coordenação e tomadas de decisão. $\mathrm{Na}$ categoria "Atividades e equipamentos", com menor incidência de fatores

90 Muianga, E. A. D.; Granja, A. D.; Ruiz, J. de A. 
responsáveis pelos desvios do que a de "Gerenciamento", foi frequente a menção a aspectos como escassez e falta de qualificação de mão de obra, equipamentos precários e em quantidade insuficiente, problemas com qualidade e consequente retrabalho. As categorias de "Financiamento", "Aspectos ambientais e econômicos" e "Projeto e documentação" também seguem apresentando relevância. Fatores relacionados a provisionamento de recursos financeiros necessitam maior atenção, devido a problemas de escassez e indisponibilidade de recursos. Por exemplo, em relação aos recursos financeiros, os fatores mais frequentes são a forma de pagamento apenas após o escopo completo, dificuldades na quitação de financiamentos pelas construtoras e dificuldades e habilidades financeiras precárias do proprietário. Em relação à categoria "Aspectos ambientais e econômicos", a falta de uma investigação mais aprofundada do solo, a falta de uma previsão das condições climáticas e as condições socioeconômicas são os fatores mais decorrentes nos empreendimentos. Erros e omissões e a qualidade do projeto foram os fatores mais citados na categoria "Projeto e documentação". Como consequência dos problemas do processo de projeto, têm-se as alterações e aumento do escopo do empreendimento, na forma de trabalhos adicionais.

Por último, as categorias "Organização", "Contratos" e "Relações governamentais" tiveram menos relevância. Os problemas mais comuns na "Organização" são falta de coordenação de diferentes tarefas entre diferentes subempreiteiros, falta de conhecimento e experiência em relação ao empreendimento e má liderança. Entretanto, na categoria de "Contratos" e "Relações governamentais", fatores como duração e valor irreal, pressão do governo e situação política de um país,e obtenção de permissões legais e alteração de regras governamentais foram os mais citados para a ocorrência de desvios de custos e prazos em empreendimentos.

\section{Conclusões}

A contribuição principal desta pesquisa foi sintetizar os argumentos teóricos e os resultados de um bloco multifacetado de literatura, composto de 92 estudos primários no tema desvios de custos e prazos em empreendimentos da construção civil. Com base na RSL realizada, propuseram-senove categorias:
(a) relações governamentais;
(b) contratos; organização;
(c) gerenciamento; financiamento;
(d) projeto e documentação;
(e) alterações de escopo;
(f) aspectos ambientais e econômicos; e
(g) atividades e equipamentos.

Dentro dessas categorias, foram distribuídos 95 fatores que influenciam os desvios em empreendimentos da construção civil. Os fatores tiveram de ser estratificados a partir de seus significados nos estudos primários levantados, uma vez que houve diversidade de uso de terminologias pelos diferentes autores, o que se constituiu em uma contribuição adicional desta pesquisa para o alinhamento da terminologia dentro da temática.

\section{Figura 4 - Quantidade (\%) de fatores responsáveis por desvios de custos e prazos em cada uma das categorias}

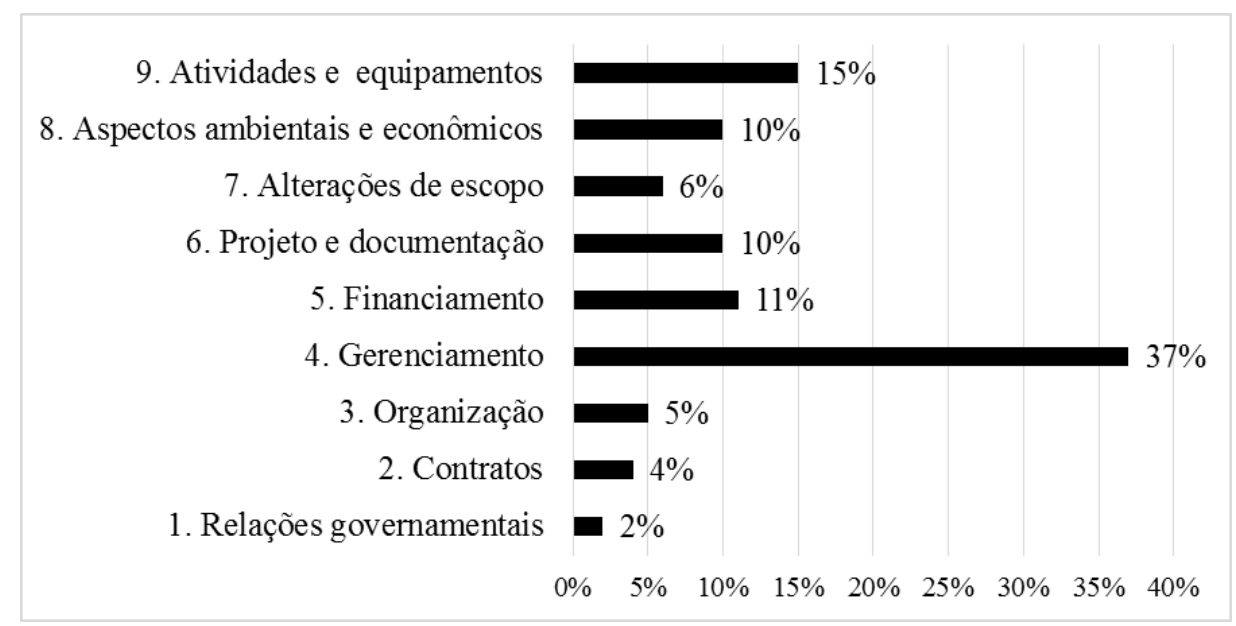


Tendo em vista as fontes primárias consultadas, analisou-se a distribuição dos fatores nas categorias. Nesse sentido, algumas categorias demonstraram maior relevância em relação a outras para a ocorrência dos desvios. É importante ressaltar que fatores relacionados à categoria "Gerenciamento", de uma forma geral, foram os mais recorrentes.

Com base apenas nos poucos estudos nacionais considerados na pesquisa, há evidências contrastantes que merecem reflexão. A categoria "Contratos" apresentou menor relevância no corpo de pesquisas consultadas quando comparada ao contexto brasileiro, isoladamente (RICARDINO; SILVA; ALENCAR, 2013). Conforme Ricardino, Silva e Alencar (2013), o risco associado à formação de preço decorre da qualidade da informação disponível em relação ao escopo de trabalho a ser executado, especialmente em contratos por preço global. Assim, especificações detalhadas sobre o produto tendem a ocasionar menores desvios esperados sobre o orçamento de referência. Por outro lado, os contratos são celebrados em fases iniciais do ciclo de vida dos empreendimentos, em que há grandes incertezas e precariedade de informação, fato que pode explicar a ocorrência frequente de alterações de escopo nos empreendimentos do setor.

As principais limitações desta pesquisa estão relacionadas à condução da RSL em si. O julgamento da qualidade dos estudos e os critérios de inclusão e exclusão das fontes primárias são etapas de difícil tomada de decisão na condução de qualquer RSL. Além disso, as diferentes pesquisas analisadas utilizaram os mais diversos métodos de abordagem na temática, o que dificultou o processo. Ainda assim, a síntese obtida a partir das fontes primárias analisadas oferece uma perspectiva nova e mais detalhada sobre o assunto, a qual não seria possível de ser obtida a partir dos resultados isolados de cada uma dessas pesquisas. Finalmente, a presente pesquisa enseja grande quantidade de desdobramentos de estudos individualizados de fatores específicos e suas correlações com desvios, especificamente para o contexto nacional.

\section{Referências}

ABD EL-RAZEK, M.; BASSIONI, H.; MOBARAK, A. Causes of Delay in Building Construction Projects in Egypt.Journal of Construction Engineering and Management, v. 134, n. 11, p. 831-841, 2008.
ADNAN, E.; JOMAH, A.-N.; MOHAN, K. Delays and Cost Overruns in the Construction Projects in the Gaza Strip. Journal of Financial

Management of Property and Construction, v. 14, p. 126-151, 2009.

AHMED, S. et al. Construction Delays in Florida: an empirical study. In: ASC ANNUAL

CONFERENCE, 39., South Carolina,

2003.Proceedings...South Carolina, 2003.

AHOMIDAN, A. Factors Affecting cost Overrun in Road Construction Projects in Saudi Arabia. International Journal of Civil \& Environmental Engineering, v. 13, n. 3, 2013.

AKINTOYE, A. S.; MACLEOD, M. J. Risk

Analysis and Management in Construction.International Journal of Project Management, v. 15, n. 1, p. 31-38, 1997.

ALAGHBARI, W. et al. The Significant Factors Causing Delay of Building construction Projects in Malaysia. Engineering, Construction and Architectural Management, v. 14, n. 2, p. 192206, 2007.

ALINAITWE, H.; MWAKALI, J. A.; HANSSON, B. Factors Affecting Productivity of Building Craftsmen: a case of Uganda. In: MWAKALI, J. A.; TABAN-WANI, G. (Orgs.). PROCEEDINGS FROM THE INTERNATIONAL CONFERENCE ON ADVANCES IN ENGINEERING AND TECHNOLOGY, Oxford, 2006. Proceedings... Oxford: Elsevier Science, 2006.

AL-MOMANI, A. H. Construction Delay: a quantitative analysis. International Journal of Project Management, v. 18, n. 1, p. 51-59, 2000.

AL-NAJJAR, J. M. Factors Influencing Time and Cost Overruns on Construction Projects in the Gaza Strip. Master, Gaza: The Islamic University of Gaza, 2008.

ARDITI, D.; AKAN, G. T.; GURDAMAR, S. Cost Overruns in Public Projects.International Journal of Project Management, v. 3, n. 4, p. 218-224, 1985

ASSAF, S. A.; AL-HEJJI, S. Causes of Delay in Large Construction Projects. International Journal of Project Management, v. 24, n. 4, p. 349-357, 2006.

ASSAF, S.; AL-KHALIL, M.; AL-HAZMI, M. Causes of Delay in Large Building Construction Projects. Journal of Management in

Engineering, v. 11, n. 2, p. 45-50, 1995.

AZIZ, R. F. Factors Causing Cost Variation ForConstructing Wastewater Projects in Egypt. Alexandria Engineering Journal, v. 52, n. 1, p. 51-66, 2013a. 
AZIZ, R. F. Ranking of Delay Factors in Construction Projects after Egyptian Revolution. Alexandria Engineering Journal, v. 52, n. 3, p. 387-406, 2013b.

BIERNACKI, P.; WALDORF, D. Snowball Sampling: problems and techniques of chain referral sampling. Sociological Methods \& Research, v. 10, n. 2, p. 141-163, 1981.

BOMBIG, A.; GORCZESKI, A. P. E. V. Por Que Tudo atrasa no Brasil. 2013. Disponível em: <http://revistaepoca.globo.com/tempo/noticia/2013 /05/por-que-tudo-atrasa-no-brasil.html $>$. Acesso em: 26 jan. 2015.

BRERETON, O. P. et al. Lessons FromApplying the Systematic Literature Review Process Within the Software Engineering Domain. The Journal of Systems and Software, v. 80, n. 4, p. 571-583, 2007.

CHAN, D. W. M.; KUMARASWAMY, M. M.An Evaluation of Construction Time Performance in the Building Industry.Building and Environment, v. 31, n. 6, p. 569-578, 1996.

CHAN, D. W.; KUMARASWAMY, M. M. A Comparative Study of Causes of Time Overruns in Hong Kong Construction Projects. International Journal of Project Management, v. 15, n. 1, p. 55-63, 1997.

COUTO, J.P. Study and analysis of the reasons for the lack of competitiveness of Portuguese construction industry: implemented research surveys. In: IPMA WORLD CONGRESS ON PROJECT MANAGEMENT, 20, Shanghai, 2006. Anais... Shangai, 2006.

CREEDY, G. D. Risk Factors Leading to Cost Overrun in the Delivery of Highway Construction Projects. Queensland, 2006.Thesis Queensland University of Technology, Queensland, 2006.

DLAKWA, M.; CULPIN, M. F. Reasons ForOverrun in Public Sector Construction Projects in Nigeria. International Journal of Project Management, v. 8, n. 4, p. 237-241, 1990.

DOLOI, H. et al. Analyzing Factors Affecting Delays in Indian Construction Projects. International Journal of Project Management, v. 30, n. 4, p. 479-489, 2012.

DOMINIC, A.-D. D.; SMITH, S. D. Rethinking Construction Cost Overruns: cognition, learning and estimation. Journal of Financial Management of Property and Construction, v. 19, n. 1, p. 38-54, 2014.
ELINWA, A.; BUBA, S. Construction Cost Factors in Nigeria. Journal of Construction Engineering and Management, v. 119, n. 4, p. 698-713, 1993.

ENSHASSI, A.; ARAIN, F.; AL-RAEE, S. Causes of Variation Orders in Construction Projects in the Gaza Strip. Journal of Civil Engineering and Management, v. 16, p. 540-551, 2010.

ENSHASSI, A.; KUMARASWAMY, M.; JOMAH, A.-N. Significant Factors Causing Time and Cost Overruns in Construction Projects in the Gaza Strip: contractors' perspective. International Journal of Construction Management, v. 10, n. 1, p. 35-60, 2010.

FALLAHNEJAD, M. H. Delay Causes in Iran Gas Pipeline Projects. International Journal of Project Management, v. 31, n. 1, p. 136-146, 2013.

FELDMAN. Why Categorize? Kmworld, v. 13, n. 9, 2004.

FRIMPONG, Y.; OLUWOYE, J.; CRAWFORD, L. Causes of Delay and Cost Overruns in Construction of Groundwater Projects in a Developing countries: Ghana as a case study. International Journal of Project Management, v. 21, n. 5, p. 321-326, 2003.

FUGAR, F. D. K.; AGYAKWAH-BAAH, A. B. Delays in Building Construction Projects in Ghana.Australasian Journal of Construction Economics and Building, v. 10, n. 1/2, p. 103116, 2010.

GONZÁLEZ, P. et al. Analysis of Causes of Delay and Time Performance in Construction Projects. Journal of Construction Engineering and Management, v. 140, n. 1, 2014.

HAMZAH, N. et al. Cause of Construction Delay: theoretical framework. Procedia Engineering, v. 20, p. 490-495, 2011.

HAQ, Y.; ASLAM, M. Causes of Delay in Construction Projects of Punjab-Pakistan: an empirical study. Journal of Basic and Applied Scientific Research, v. 3, n. 10, p. 87-96, 2013.

HWANG, B.-G.; ZHAO, X.; NG, S. Y. Identifying the Critical Factors Affecting Schedule Performance of Public Housing Projects. Habitat International, v. 38, p. 214-221, 2013.

IYER, K. C.; JHA, K. N. Factors Affecting Cost Performance: evidence from Indian construction projects. International Journal of Project Management, v. 23, n. 4, p. 283 295, 2005. 
IYER, K.; JHA, K. Critical Factors Affecting Schedule Performance: evidence from Indian construction projects. Journal of Construction Engineering and Management, v. 132, n. 8, p. 871-881, 2006.

JAHANGER, Q. Important Causes of Delay in Construction Projects in Baghdad City. Australian Journal of Basic and Applied Sciences, v. 7, n. 4, p. 14-23, 2013.

JOSEPHSON, P. -E.; HAMMARLUND, Y. The Causes and Costs of Defects in Construction: a study of seven building projects. Automation in Construction, v. 8, n. 6, p. 681-687, 1999.

KADIR, M. R. A. et al. Factors Affecting Construction LabourProductivity For Malaysian Residential Projects. Structural Survey, v. 23, n. 1, p. 42-54, 2005.

KALIBA, C.; MUYA, M.; MUMBA, K. Cost Escalation and Schedule Delays in Road Construction Projects in Zambia. International Journal of Project Management, v. 27, n. 5, p. 522-531, 2009.

KAMANGA, M. J.; STEYN, W. J. von der. Causes of Delay in Road Construction Projects in Malawi. Journal of the South African Institution of Civil Engineering, v. 55, n. 3, p. 79-85, 2013.

KAMING, P. F. et al. Factors Influencing Construction Time and Cost Overruns on HighRise Projects in Indonesia. Construction Management and Economics, v. 15, n. 1, p. 8394, 1997a.

KAMING, P. F. et al. Factors Influencing Craftsmen's Productivity in Indonesia. International Journal of Project Management, v. 15 , n. 1 , p. 21-30, 1997 b.

KITCHENHAM, B. et al. Systematic Literature Reviews in Software Engineering: a systematic literature review. Information and Software Technology, v. 51, n. 1, p. 7-15, 2009.

KOUSHKI, P. A.; AL-RASHID, K.; KARTAM, N. Delays and Cost Increases in the Construction of Private Residential Projects in Kuwait.

Construction Management and Economics, v. 23, n. 3, p. 285-294, 2005.

KOUSHKI, P. A.; KARTAM, N. Impact of Construction Materials on Project Time and Cost in Kuwait. Engineering, Construction and Architectural Management, v. 11, n. 2, p. 126132, 2004.
LEE, J.-K. Cost Overrun and Cause in Korean Social Overhead Capital Projects: roads, rails, airports, and ports. Journal of Urban Planning and Development, v. 134, n. 2, p. 59-62, 2008.

LE-HOAI, L.; LEE, Y. D.; LEE, J. Y. Delay and Cost Overruns in Vietnam Large Construction Projects: a comparison with other selected countries. Ksce Journal of Civil Engineering, v. 12 , n. 6 , p. $367-377,2008$

LO, T.; FUNG, I.; TUNG, K. Construction Delays in Hong Kong Civil Engineering Projects. Journal of Construction Engineering and Management, v. 132, n. 6, p. 636-649, 2006.

LONG, N. D. et al. Large Construction Pprojects in Developing Countries: a case study from Vietnam. International Journal of Project Management, v. 22, n. 7, p. 553-561, 2004.

LOVE, P. et al. Rework in Civil Infrastructure Orojects: determination of cost predictors. Journal of Construction Engineering and Management, v. 136, n. 3, p. 275-282, 2010.

MAHAMID, I. Common Risks Affecting Time Overrun in Road Construction Projects in Palestine: contractors' perspective. Australasian Journal of Construction Economics and Building, v. 13, n. 2, p. 45-53, 2013.

MAHAMID, I.; BRULAND, A.; DMAIDI, N. Causes of Delay in Road Construction Projects.Journal of Management in Engineering, v. 28, n. 3, p. 300-310, 2012.

MAKOVŠEK, D.; TOMINC, P.; LOGOŽAR, K.A Cost Performance Analysis of Transport Infrastructure Construction in Slovenia.Transportation, v. 39, n. 1, p. 197-214, 2012.

MANAVAZHI, M. R.; ADHIKARI, D. K. Material and Equipment Procurement Delays in Highway Projects in Nepal. International Journal of Project Management, v. 20, n. 8, p. 627-632, 2002.

MAÑELELE, I.; MUYA, M. Risk Identification on Community-Based Construction Projects in Zambia.Journal of Engineering, Design and Technology, v. 6, n. 2, p. 145-161, 2008.

MANSFIELD, N.; UGWU, O.; DORAN, T. Causes of Delay and Cost Overruns in Nigerian Construction Projects. International Journal of Project Management, v. 12, n. 4, p. 254-260, 1994.

MARZOUK, M. M.; EL-RASAS, T. I. Analyzing Delay Causes in Egyptian Construction

Projects.Journal of Advanced Research, v. 5, n. 1, p. 49-55, 2014. 
MEMON, A. H.; RAHMAN, I. A.; AZIS, A. A. Preliminary Study on Causative Factors Leading to Construction Cost Overrun.International Journal of Sustainable Construction Engineering and Technology, v. 2, n. 1, p. 57-71, 2011.

MOTALEB, O.; KISHK, M. An Investigation IntoCauses and Effects of Construction Delays in UAE. In: ANNUAL CONFERENCE OF THE ASSOCIATION OF RESEARCHERS IN CONSTRUCTION MANAGEMENT,26., Leeds, 2010.Proceedings...Leeds, 2010.

MULROW, C. D. Rationale ForSystematic Reviews. British Medical Journal, v. 309, n. 6954, p. 597-599, 1994.

MURRAY, M.; SEIF, M. Causes of Project Delays in Nigerian Construction Industry.European Journal of Civil Engineering and Architecture, v. 10, n. 1, 2013.

MUYA, M. et al. Cost Escalation, Schedule Overruns and Quality Shortfalls on Construction Projects: the case of Zambia. International Journal of Construction Management, v. 13, p. 53-68, 2013.

NAJAFABADI, E.; PIMPLIKAR.The Significant Causes and Effects of Delays in Ghadir 2206 Residential Project. Journal of Mechanical and Civil Engineering, v. 7, n. 4, p. 75-81, 2013.

NEGA, F. Causes and Effects of Cost Overrun on Public Building Construction Projects in Ethiopia. 2008. Master Thesis - Addis Ababa University, Addis Ababa, Ethiopia. 2008.

NUTAKOR, G. Assessing Final Cost of Construction at Bid Time. Cost Engineering (Morgantown, West Virginia), v. 49, n. 11, p. 1016, 2007.

ODEH, A. M.; BATTAINEH, H. T. Causes of Construction Delay: traditional contracts. International Journal of Project Management, v. 20 , n. 1, p. 67-73, 2002.

OGUNLANA, S. O.; OLOMOLAIYE, P. O.A Survey of Site Management Practice on Some Selected Sites in Nigeria. Building and Environment, v. 24, n. 2, p. 191-196, 1989.

OGUNLANA, S. O.; PROMKUNTONG, K.; JEARKJIRM, V. Construction Delays in a FastGrowing Economy: comparing Thailand with other economies. International Journal of Project Management, v. 14, n. 1, p. 37-45, 1996.

OKPALA, D.; ANIEKWU, A. Causes of High Costs of Construction in Nigeria. Journal of Construction Engineering and Management, v. 114, p. 233-244, 1988.
OLADAPO, A. A. A quantitative Assessment of the Cost and Time Impact of Variation Orders on Construction Projects. Journal of Engineering, Design and Technology, v. 5, n. 1, p. 35-48, 2007. OLAWALE, Y. A.; SUN, M. Cost and Time Control of Construction Projects: inhibiting factors and mitigating measures in practice. Construction Management and Economics, v. 28, n. 5, p. 509526, 2010.

ORANGI, A.; PALANEESWARAN, E.; WILSON, J. Exploring Delays in Victoria-Based Australian Pipeline Projects.Procedia Engineering, v. 14, p. 874-881, 2011.

PARK, Y.-I.; PAPADOPOULOU, T. C. Causes of Cost Overruns in Transport Infrastructure Projects in Asia: their significance and relationship with project size. Built Environment Project and Asset Management, v. 2, n. 2, p. 195-216, 2012.

PETTICREW, M. Systematic Reviews in the Social Sciences: a practical guide. Malden, Mass: [Blackwell Publ.], 2009.

PIRES, B.; TEIXEIRA, J. M. C.; MOURA, H. M. P. Management Functions and Competitiveness in the Portuguese Construction Industry.In: CME 25 CONFERENCE CONSTRUCTION MANAGEMENT AND ECONOMICS "PAST, PRESENT AND FUTURE”, 2007. Proceedings... 2007.

POTTY, N. S.; IRDUS, A. B.; RAMANATHAN, C. Case Study and Survey on Time and Cost Overrun of Multiple D\&B Projects.In: NATIONAL POSTGRADUATE CONFERENCE (NPC), 2011. Proceedings... 2011.

POURROSTAM, T.; ISMAIL, A. Causes and Effects of Delay in Iranian Construction Projects. International Journal of Engineering and Technology, v. 4, n. 5, oct.2012.

RAHMAN, I. A. et al. Time and Cost Performance of Construction Projects in Southern and Central Regions of Peninsular Malaysia. In: IEEE COLLOQUIUM ON HUMANITIES, SCIENCE AND ENGINEERING (CHUSER), 2012.Proceedings... 2012.

RAHMAN, I. A.; MEMON, A. H.; KARIM, A. T. A. Relationship BetweenFactors of Construction Resources Affecting Project Cost. Modern Applied Science, v. 7, n. 1, p. 67-75, $2013 \mathrm{~b}$.

RAHMAN, I. A.; MEMON, A. H.; KARIM, A. T. A. Significant Factors Causing Cost Overruns in Large Construction Projects in Malaysia. Journal of Applied Sciences, v. 13, n. 2, p. 286-293, 2013a. 
RAMANATHAN, C.; POTTY, N. S.; BIN IDRUS, A. Risk factors influencing time and cost overrun in multiple D\&B projects in Malaysia: a case study. In: IEEE INTERNATIONAL CONFERENCE ON INDUSTRIAL ENGINEERING AND ENGINEERING MANAGEMENT, Singapore, 2011. Anais... Singapore, 2011.

RAMANATHAN, C.; NARAYANAN, S. P.; IDRUS, A. B. Construction Delays Causing Risks on Time and Cost: a critical review. Australasian Journal of Construction Economics and Building, v. 12, n. 1, p. 37-57, 2012.

REN, Z.; ATOUT, M.; JONES, J. Root Causes of Construction Project Delays in Dubai. In: PANNUAL ARCOM CONFERENCE, 24., Cardiff, UK, 2008. Proceedings... Cardiff, UK, 2008.

RIBEIRO, P.et al. Success Evaluation Factors in Construction Project Management: some evidence from medium and large Portuguese companies.

Ksce Journal of Civil Engineering, v. 17, n. 4, p. 603-609, 2013.

RICARDINO, R.; SILVA, S.; ALENCAR, C. Causas Frequentes de Reivindicações Contratuais. Revista Engenharia, maio a julho, 2013, ano 70, n. 615, p. 90-94, 2013.

ROACHANAKANAN, K. A Case Study of Cost Overruns in a Thai Condominium

Project.Texas, 2005. Ph.D. (Dissertation) - Texas A\&M University, Texas, 2005.

ROSENFELD, Y. Root-Cause Analysis of Construction-Cost Overruns. Journal of Construction Engineering and Management, v. 140, n. 1, p. 04013039, 2014.

ROWLAND, H. J. The Causes and Effects of Change Orders on the Construction

Process.Georgia: Georgia Institute of Technology, 1981.

SAMBASIVAN, M.; SOON, Y. W. Causes and Effects of Delays in Malaysian Construction Industry. International Journal of Project Management, v. 25, n. 5, p. 517-526, 2007.

SEMPLE, C.; HARTMAN, F. T.; JERGEAS, G. Construction Claims and Disputes: causes and cost/time overruns. Journal of Construction Engineering and Management, v. 120, n. 4, p. 785-795, 1994.

SHANE, J. et al. Construction Project Cost Escalation Factors. Journal of Management in Engineering, v. 25, n. 4, p. 221-229, 2009.
SHEHU, Z.; ENDUT, I. R.; AKINTOYE, A. Factors Contributing to Project Time and Hence Cost Overrun in the Malaysian Construction Industry. Journal of Financial Management of Property and Construction, v. 19, n. 1, p. 55-75, 2014.

SOEKIMAN, A. et al. Factors Relating to Labor Productivity Affecting the Project Schedule Performance in Indonesia. Procedia Engineering, v. 14, n. 10, p. 865-873, 2011.

SUGIHARTO, A.; HAMPSON, K. Identifying the Important Causes of Delays in Building Construction Projects.In: EAST ASIA-PACIFIC CONFERENCE ON STRUCTURAL ENGINEERING AND CONSTRUCTION, 9., Indonesia, 2003. Proceedings...Indonesia, 2003.

SUNDAY, O. A. Impact of Variation Orders on Public Construction Projects. In: ANNUAL CONFERENCE ASSOCIATION OF RESEARCHERS IN CONSTRUCTION MANAGEMENT, 26., Leeds, 2010.Proceedings...Leeds, 2010.

SWEIS, G. et al. Delays in Construction Projects: the case of Jordan. International Journal of Project Management, v. 26, n. 6, p. 665-674, 2008.

TRANFIELD, D.; DENYER, D.; SMART, P. Towards a Methodology For Developing Evidence-Informed Management Knowledge by Means of Systematic Review. British Journal of Management, v. 14, n. 3, p. 207-222, 2003.

VIDALIS, M.; NAJAFI, T. Cost and Time Overruns in Highway Construction. Canadá, 2002.

WANG, J.; YUAN, H. Factors Affecting Contractors' Risk Attitudes in Construction Projects: case study from China. International Journal of Project Management, v. 29, n. 2, p. 209-219, 2011.

WIGUNA, I. P. A.; SCOTT, S. Nature of the Critical Risk Factors Affecting Project Performance in Indonesian Building Contracts.In: ANNUAL CONFERENCE ASSOCIATION OF RESEARCHERS IN CONSTRUCTION MANAGEMENT, 21.,2005.Proceedings... 2005.

ZHU, K.; LI, L. A Stage-By-Stage Factor Control Framework ForCost Estimation of Construction Projects. In: CLIENTS DRIVING INNOVATION INTERNATIONAL CONFERENCE, Brisbane, 2004. Proceedings...Austrália: CIB, 2004. 
ZOU, P. X. W.; ZHANG, G.; WANG, J. Understanding the Key Risks in Construction Projects in China. International Journal of Project Management, v. 25, n. 6, p. 601-614, 2007.

\section{Agradecimentos}

Agradecimentos à CAPES, pela concessão de bolsa a um dos autores desta pesquisa.

Elisa Atália Daniel Muianga

Laboratório de Gerenciamento na Construção, Departamento de Arquitetura e Construção | Universidade Estadual de Campinas e Asna Construções e Engenharia | Rua do Tchamba, Coop | Maputo - Moçambique | 356 | Tel.: (258) 2149-3603 |

E-mail: elisa.atalia@gmail.com

\section{Ariovaldo Denis Granja}

Laboratório de Gerenciamento na Construção, Departamento de Arquitetura e Construção | Universidade Estadual de Campinas | Av. Albert Einstein, 951, Cidade Universitária Zeferino Vaz, Barão Geraldo | Campinas - SP - Brasil | Caixa-Postal 6021 | CEP 13083-852 | Tel.: (19) 3521-2082 | E-mail: adgranja@fec.unicamp.br

\section{Joyce de Andrade Ruiz}

Laboratório de Gerenciamento na Construção, Departamento de Arquitetura e Construção | Universidade Estadual de Campinas | E-mail: joyce.a.r@live.com

\section{Revista Ambiente Construído}

Associação Nacional de Tecnologia do Ambiente Construído

Av. Osvaldo Aranha, $99-3^{\circ}$ andar, Centro

Porto Alegre - RS - Brasil

CEP $90035-190$

Telefone: +55 (51) 3308-4084

Fax: +55 (51) 3308-4054

www.seer.ufrgs.br/ambienteconstruido

E-mail: ambienteconstruido@ufrgs.br 